\title{
Monnaie de compte et monnaie réelle : des relations mal étudiées
}

\section{Philippe Lardin}

\section{(2) OpenEdition}

1 Journals

Édition électronique

URL : https://journals.openedition.org/ress/208

DOI : $10.4000 /$ ress.208

ISSN : 1663-4446

Éditeur

Librairie Droz

Édition imprimée

Date de publication : 1 juillet 2007

Pagination : 45-68

ISBN : 978-2-600-01155-6

ISSN : 0048-8046

Référence électronique

Philippe Lardin, "Monnaie de compte et monnaie réelle : des relations mal étudiées », Revue européenne des sciences sociales [En ligne], XLV-137 | 2007, mis en ligne le 01 juillet 2010, consulté le 21 septembre 2021. URL : http://journals.openedition.org/ress/208 ; DOI : https://doi.org/10.4000/ ress. 208 


\section{Philippe LARDIN}

\section{MONNAIE DE COMPTE ET MONNAIE RÉELLE: DES RELATIONS MAL ÉTUDIÉES}

Les comptabilités qui nous sont parvenues - essentiellement des deux derniers siècles du Moyen Age - ont longtemps été considérées par les historiens comme des sources de première importance pour mener à bien une étude statistique des réalités économiques de cette période. Pour la Normandie orientale, Guy Bois a ainsi pu estimer qu'il avait rassemblé, à partir de la plupart des comptabilités royales et ecclésiastiques conservées à Rouen ou à la Bibliothèque Nationale de France, "assez d'indications chiffrées pour qu' une étude quantitative soit entreprise $»^{1}$ et il a cru pouvoir dresser des tableaux (cf. tableau 1), apparemment indiscutables, enregistrant année après année les salaires des ouvriers spécialisés et des

Tableau I: Monnaies en circulation à Rouen en 1421-1422

\begin{tabular}{|c|c|c|}
\hline Nom de la pièce & Valeur légale & Nombre de mentions \\
\hline $\begin{array}{l}\text { Monnaies anglaises } \\
\text { Noble d'or } \\
\text { Esterlin }\end{array}$ & $\begin{array}{l}45 \text { sous } \\
\text { Monnaie de compte }\end{array}$ & $\begin{array}{l}99 \\
21\end{array}$ \\
\hline $\begin{array}{l}\text { Monnaies «françaises» } \\
\text { Ecu d'or (Vieil or) } \\
\text { Ecu d'or } \\
\text { Ecu d'or «à la couronne» } \\
\text { Mouton d'or } \\
\text { Petit mouton } \\
\text { Franc } \\
\text { Salut d'or } \\
\text { Blanc } \\
\text { Petit blanc } \\
\text { Double nouveau } \\
\text { Gros }\end{array}$ & $\begin{array}{c}37 \text { sous } 6 \mathrm{~d} ; 35 \text { sous ; } \\
27 \text { sous } 6 \text { deniers } ; 22 \text { sous } 6 \mathrm{~d} \text {. } \\
22 \text { sous } 6 \text { deniers } \\
22 \text { sous } 6 \text { deniers } \\
15 \text { sous } \\
15 \text { sous } \\
? \\
25 \text { sous } \\
10 \text { deniers } \\
5 \text { deniers } \\
2 \text { deniers } \\
20 \text { deniers } \\
\text { jusqu'au } 12 \text { avril } 1421 \\
5 \text { deniers du } 12 \text { avril } \\
\text { au } 30 \text { novembre } \\
2 \text { deniers obole à partir } \\
\text { du } 1^{\text {er }} \text { décembre } \\
\text { démonétisé en mai } 1422\end{array}$ & $\begin{array}{c}325 \\
14 \\
32 \\
7 \\
2 \\
19 \\
10 \\
3 \\
36 \\
818\end{array}$ \\
\hline
\end{tabular}

\footnotetext{
1 Bois, 1976, p. 92.
} 
manœuvres du secteur du bâtiment, ainsi que des graphiques montrant l'évolution du prix de diverses céréales ou de certains matériaux de construction. Bien sûr, afin de pouvoir éliminer les effets des modifications de la valeur de la monnaie, il n'avait pas négligé de «déflater» toutes ces données en les exprimant en quantité de céréales, notamment en froment. Pourtant, à aucun moment, il ne se posa la question de savoir si les données dont il se servait, exprimées en monnaie de compte, autrement dit en monnaie virtuelle et, par conséquent, totalement inexistante, subissaient les conséquences des évolutions de la valeur des pièces en circulation, c'est-à-dire de la monnaie réelle dont les changeurs exprimaient le prix lors de certaines transactions, mais dont les autorités fixaient une valeur légale nécessairement acceptée par la plupart des utilisateurs.

C'est donc sur les problèmes posés par les relations entre la monnaie de compte utilisée par les rédacteurs de comptabilités et les réalités monétaires basées sur la valeur légale des pièces en circulation que je voudrais attirer l'attention dans cette communication. Pour cela, j'utiliserai des données normandes déjà utilisées le plus souvent par Guy Bois, mais en les replaçant dans le contexte de trois périodes distinctes : la crise monétaire de 1420-1422, la fin de la guerre de Cent Ans et la fin des années 1460, pendant lesquelles se firent sentir en Normandie les conséquences de ce qu'on a appelé la Ligue du Bien Public. Les quelques remarques qui vont suivre sont les prémices d'un travail en cours sur les pièces en circulation d'après les sources écrites et ne sont, pour l'instant, que des directions de travail.

La crise monétaire des années 1420-1422 a beaucoup frappé les esprits. Aussi bien ceux des contemporains que ceux des historiens actuels. La hausse des prix à première vue vertigineuse - qui eut lieu alors toucha l'ensemble du royaume de France mais elle eut en Normandie des aspects particuliers du fait de la victoire anglaise qui venait de se manifester par la prise de Rouen ${ }^{2}$ et la chute consécutive de la presque totalité des places fortes de la province ${ }^{3}$. En effet, la ponction représentée par la composition de 400000 livres que le roi d'Angleterre Henry V imposa à Rouen s'ajoutant aux difficultés dues à la guerre, ainsi que l'entrée dans la province de monnaies royales ou delphinales françaises et de plusieurs autres monnaies locales, se traduisirent assez vite par des troubles monétaires considérables qui constituèrent une gêne importante pour les milieux économiques normands aussi bien que pour le souverain anglais ${ }^{4}$.

Le fait apparaît de manière plus ou moins nette au fil des chroniques ou encore des comptabilités, en particulier dans un compte de la fabrique de la cathédrale de Rouen $^{5}$. Toutefois, les importantes lacunes des années suivantes ne permettent pas de faire un examen véritablement approfondi du phénomène. Les registres du

2. Sur l'histoire monétaire en général, voir la bibliographie en fin d'article.

3 Sur la situation de la Normandie entre 1410 et 1422, voir Bois, 1976, pp. 284-293 ; C.T. Allmand, 1987.

4 Dès le 12 janvier 1420 (n. st.), le roi Henry V se plaignait de l'entrée en Normandie de monnaies françaises d'or et d'argent, lesquelles sont moult foibles, tant en pois comme en loy. F. de Saulcy Paris, 1878, p. 7. Le 20 novembre 1421, il mettait en cause les grandes frauldes, mauvaisies et deceptions de celui qui se dit dauphin. Ibid. p. 13.

5 Archives Départementales de Seine-Maritime (dans la suite ADSM), G2486. 
tabellionage de Rouen qui couvrent sans interruption, jour après jour, la période qui va de 1419 à 1423, fournissent, en revanche, une information continue et relativement précise ${ }^{6}$. Ces registres ne disent que peu de choses sur la valeur réelle titre ou poids - des pièces en circulation au cours de cette période. Ils nous mettent en présence de monnaies aux noms précis, dont le cours est fixé officiellement par les autorités, mais dont la valeur intrinsèque reste incertaine. Il n'est donc pas possible, à partir de telles sources, de savoir précisément comment la valeur réelle des pièces en circulation, estimée par les changeurs, évolua au cours de ces années de crise mais ce n'est pas forcément le plus intéressant. En fait, ces documents nous permettent de saisir la manière dont les Normands réagirent aux troubles monétaires auxquels ils étaient confrontés. Ils nous permettent surtout, ce qui est l'essentiel pour le sujet qui nous occupe, de décrire les conséquences de la baisse de la valeur des pièces en circulation sur les prix et sur certains salaires, avant d'essayer de décrire les effets des bouleversements de la monnaie réelle sur les données en monnaie de compte.

La crise de 1420-1422 fut avant tout une crise du gros. C'était alors, en effet, la pièce la plus fréquemment employée dans les transactions et celle dont la valeur fut le plus affectée par la situation monétaire ${ }^{7}$. Au début de la période que nous étudions, sa valeur légale était de 20 deniers $^{8}$. Les gros, contrairement à ce que disent les ouvrages de numismatique ${ }^{9}$, ne sont jamais appelés florettes dans les documents normands qui nous sont parvenus, mais parfois seulement royaulx ${ }^{10}$. Pour des raisons qui demeurent encore obscures, mais sans doute à cause d'un contexte politique troublé et de l'émission de pièces dépréciées par le dauphin ou le duc de Bretagne aussi bien que par des faux-monnayeurs, cette pièce se déprécia considérablement au cours de l'année $1419^{11}$. Dans un premier temps, le roi Henry $\mathrm{V}$ intervint en cherchant à remettre en circulation des pièces de bon poids et de bon aloi. Le 12 janvier 1420, il fit forger de nouveaux gros auxquels il garda la valeur officielle de 20 deniers tout en en précisant minutieusement les caractéristiques, de poids, d'aloi et de figure ${ }^{12}$. Le 6 mai, puis le 16 juin suivant, de nouvelles ordonnances tentèrent encore de rétablir la valeur du gros à 20 deniers $^{13}$

${ }^{6}$ ADSM 2E1/169. Dans la suite, toutes les indications de folio sans précision de cote sont issues de ce registre.

${ }^{7}$ La composition qui avait frappé Rouen était payable en or et il est probable que ce métal était devenu très rare.

${ }^{8}$ La première mention de la valeur du gros à 20 deniers (en fait, du quart de gros à 5 deniers) par le roi Henry V est du 25 septembre 1419. A cette date, le noble d'or anglais devait valoir 48 gros, soit 2 livres et le mouton d'or 30 sous. De nouvelles lettres du roi du 12 janvier 1420 (n. st.) ordonnent l'émission de nouveaux gros de 20 deniers et en fixent les caractéristiques. Même décision le 18 avril 1420. F. de Saulcy, 1878, p. 6, 7 et 9. Le 6 mai 1420 (n. st.), le roi Charles VI fait forger des gros de 20 deniers, semblables de forme à ceulx que nous faisons de present. Ordonnances des rois de France, T.XI, p.83.

9 Par exemple, Lafaurie, p. 81 (402), p. 85 (421), p. 88 (431).

${ }^{10}$ C'est dans une ordonnance du $1^{\text {er }}$ février 1420 (n.st.) qu'on trouve le nom de royaulx pour désigner les gros. F. de Saulcy, 1878, p. 8.

${ }^{11}$ C'est ce que dit le roi Henry V dans l'ordonnance datée du 1er février 1420 (n.st.). De Saulcy, 1878, p. 8. Voir aussi Feller, 1986.

12 Bibl. nat. de France, Ms français 26043 [5464].

13 De Saulcy, 1878 , p. $10-11$ et p.12. 
mais ces mesures n'eurent pas l'effet escompté et il est probable que ces gros réévalués fuirent rapidement pour être remplacés par des pièces de moindre qualité et, de ce fait, la hausse des prix se poursuivit inexorablement.

C'est pourquoi le roi - ou son conseil - décida de changer de méthode et de ramener la valeur officielle du gros à la valeur réelle des pièces en circulation. Le samedi 12 avril 1421, après disner, le gros fut officiellement déprécié à Rouen, sa valeur officielle divisée par quatre et ramenée à 5 deniers ${ }^{14}$.

Néanmoins, cette décision royale ne fut pas suffisante pour arrêter la hausse des prix et la dépréciation du gros qui continuait à être très largement utilisé dans les transactions. Il fallut donc de nouveau diviser sa valeur officielle par 2, ce qui fut crié à Rouen le $1^{\text {er }}$ décembre 1421 , environ deux heures après mydi ${ }^{15}$. Le gros ne valait donc plus désormais que 2 deniers tournois obole, ce qui se traduisait le plus souvent dans les actes rédigés par le tabellion par la formule : «des gros qui courent à présent pour deux deniers parisis». Par rapport à Pâques de cette même année, la valeur officielle du gros avait donc été divisée par $8^{16}$. Le gros fut ensuite démonétisé et la dernière mention de cette pièce dans le registre de tabellionage est du 5 mai $1422^{17}$ tandis qu'une nouvelle pièce, le «double nouveau» d'une valeur de 2 deniers était mise en circulation.

Si l'on récapitule l'évolution du gros au cours de la brève période qui nous intéresse, on peut, comme le font de nombreux actes du registre de tabellionage que nous utilisons ici, considérer qu'avant la première dépréciation, il fallait 12 gros pour obtenir une livre, 48 à partir du 12 avril 1421 et enfin 96 après le $1^{\text {er }}$ décembre ${ }^{18}$.

${ }^{14}$ Fol. 47. Le fait est jugé suffisamment important pour que le Bourgeois de Paris en fasse mention dans son journal. Pourtant, les indications qu'il fournit ne paraissent pas totalement exactes. Si la dépréciation de la valeur du gros - qu'il rapporte en deniers parisis - est exacte, il n'y a aucune mention dans le registre rouennais de nobles à 60 sous tournois ou d'écus à 30 sous ainsi qu'il l'indique. Il semble donc avoir été victime de fausses nouvelles. Celles-ci sont d'ailleurs très inquiétantes pour la population parisienne et provoquent à Paris agitation et hausse brutale des prix, parce qu'on pense que les mêmes mesures vont y être appliquées. Ce qui se produit effectivement, selon le Bourgeois, le 3 juillet suivant, mais ne touche que le gros. Journal du Bourgeois de Paris, 1990, p. 169 (305 et 306) et p. 170 (310). La décision de Charles VI se trouve en réalité dans des lettres du 21 juin 1421 qui fixent également la valeur de l'écu à 30 sous tournois et celle du mouton à 20 sous tournois. Ordonnances des rois de France, T.XI, p. 122-125.

${ }^{15}$ Fol. 251. L'ordonnance est en réalité datée du 20 novembre. De Saulcy, 1878, p. 14. Contrairement à ce qui s'était passé pour la première dépréciation du gros, c'est le roi Charles VI qui, cette fois, en donna l'exemple le 3 novembre 1421. Journal du Bourgeois de Paris, 1990, p. 173 (321). Les deux rois menaient des politiques monétaires assez voisines, ce qui s'explique par l'entente qui régnait entre eux et par le fait que la région parisienne et la Normandie étaient très liées sur le plan économique et, par conséquent, sur le plan monétaire.

${ }^{16}$ Un recueil sur les monnaies appartenant à l'abbaye de Saint-Ouen rédigé à une date inconnue indiquait que le marc d'argent était passé de 9 livres en mars 1419 à 26 livres le 28 juin 1420 et affirmait qu'il avait une valeur légale qui demeurait à 20 deniers, cette manière de compter étant ordonnée à faire iusques au premier jour de décembre. Il s'agit donc d'une information erronée qui oblige à remettre en cause la confiance que l'on pourrait accorder à ces livres de changeurs sur la rédaction desquels on ignore tout. Arch. mun. de Rouen Ms I 58, fol. 20, cité par Mollat, 1953, p. 28.

17 Fol. 398v.

${ }^{18}$ Il n'est donc pas étonnant que lors de transactions en faible monnaie, dans lesquelles on continuait à utiliser des gros dont la valeur en métal précieux était dépréciée, mais dont le montant théorique 
Ces mutations officielles de la valeur du gros modifièrent évidemment le comportement de ceux qui effectuaient des transactions. Néanmoins, l'aspect le plus remarquable que les sources font apparaître est la lenteur avec laquelle ces changements entrèrent dans les habitudes. Pendant les derniers jours du mois de mars 1421 , les actes du tabellionage ne contenaient pratiquement aucune indication sur la manière dont les paiements enregistrés avaient été ou devaient être effectués ${ }^{19}$. La crise monétaire était pourtant déjà sensible et la monnaie blanche, en particulier le gros, avait nettement commencé à se déprécier. C'est pourquoi, les utilisateurs du tabellionage faisaient insérer dans les actes des formules de protection contre d'éventuelles contestations au moment des paiements ou des remboursements. Lorsque, le 26 mars 1421, la confrérie Notre-Dame de Rouen acheta 600 livres de rente au seigneur de Saint-Pierre et de la Gaillarde, elle précisa que le rachat de cette rente était possible à condition de payer la somme nécessaire en la monnoye qui couroit lors [de la transaction] nonobstant mutacion de monnoye ${ }^{20}$. Après la première dépréciation du gros qui eut lieu, on l'a dit, le 12 avril, les clients du tabellion pouvaient réagir de plusieurs manières. Ils pouvaient immédiatement prendre en compte la décision royale et, pour faire coïncider monnaie de compte et monnaie réelle, diviser par 4 toutes les sommes correspondant à des gros. C'est ce que fit le capitaine de Bayeux le 25 novembre 1421. Alors qu'il avait reçu 6921 livres 13 sous 4 deniers en gros de 20 deniers pour payer les gages des gens d'armes qu'il avait sous ses ordres, il «signa» une quittance de 1730 livres 8 sous 4 deniers (soit quatre fois moins) en gros de 5 deniers selon la nouvelle ordonnance faicte par le Roy nostre sire ${ }^{21}$. C'est ce que fit également le seigneur de Doudeville quand il bailla son manoir à ferme pour 50 livres par an, en indiquant que la somme était comptée du pris et aloy de la monnoie nouvelle ordon$n e ́ e^{22}$. Cette nouvelle manière d'exprimer en monnaie de compte les montants des transactions aboutissait donc à une apparente baisse des sommes indiquées.

Les clients du tabellion pouvaient aussi continuer à enregistrer les transactions en monnaie de compte comme avant la dévaluation sans apparemment en tenir compte. C'est pourquoi, à partir du 15 avril, le tabellion précisa presque systématiquement, après avoir indiqué le montant de la transaction en monnaie de compte, autrement dit en livres, sous et deniers, qu'il s'agissait de telle monnoye comme il court pour le présent, a prendre chacun gros pour un petit blanc ${ }^{23}$, autrement dit pour 5 deniers. Ce faisant, les clients du tabellion entérinaient la dévaluation du gros, mais continuaient à exprimer les montants en utilisant la valeur des pièces

restait de 20 deniers, on ait parfois compté les pièces utilisées en douzaines de gros Fol. 294. Le 16 janvier 1422, une maison est vendue 20 livres de faible monnaie et elle est payée en 20 douzaines de gros qui à pasques derrain passé avoient cours pour 20 deniers.

19 Fol. 14v.

${ }^{20}$ Fol. 3v. Encore, le 23 avril 1421, une vente de 20 livres de rente entre des habitants de 2 villages de la région rouennaise fut enregistrée pour un montant de 100 livres monnoye ayans cours en caresme devant passé. Fol. 63v.

${ }^{21}$ Bibl. nat. de France, Ms français 26044 [5681].

${ }^{22}$ Fol. 55. Dans ce cas, on indique que les paiements se font en gros, à 48 gros pour livre ou même à 48 gros pour franc. Fol. 51v.

${ }^{23}$ Fol 52. 
qui avait cours auparavant. Ils inséraient de plus dans les actes une formule stéréotypée, sans doute proposée par le tabellion, précisant que le montant en monnaie de compte, s'établissait à 12 gros pour livre, des gros qui souloient courir pour 20 deniers et qui courent de present pour 5 deniers tournois.

Les clients du tabellion pouvaient aussi indiquer dans l'acte enregistré que les paiements s'étaient faits en monnaie d'or, apparemment plus stable et de plus grande valeur légale, ou bien en une autre monnaie. Ils pouvaient enfin s'abstenir de la moindre indication monétaire ou se contenter d'indiquer, comme ce couple de Rouennais qui avait prêté 50 livres à un écuyer nommé Jacques d'Orléans, que cette somme devrait être remboursée en telle monnoye qui courra au jour sainct Michiel nonobstant pois ou aloy, mutacion de monnoye, autrement dit insérer une formule de protection comme pendant la période qui précédait la dépréciation ${ }^{24}$.

Le 13 mai 1421, pour la première fois, une transaction fut exprimée par le tabellion en fieble monnoye, ce qui signifiait que le montant des paiements continuait à être exprimé en comptant 12 gros pour une livre ${ }^{25}$, mais que ces pièces avaient en réalité une valeur légale inférieure à la valeur nominale à laquelle elles auraient dû être forgées. Ainsi, on ne changeait pas le montant des sommes mises en jeu, mais leur valeur était considérée comme faible. L'utilisation de la faible monnaie devint courante et même systématique dans les semaines qui suivirent, bien qu'elle fît un peu double emploi avec la mention indiquant la valeur de compte du gros.

Les actes précisant que les transactions s'étaient faites en forte monnaie, autrement dit en comptant 48 gros $(12 \times 4)$ pour une livre-monnaie, sont plus rares. D'avril à novembre 1421, on n'en compte jamais plus de 8 par mois et leur part dans l'ensemble des actes à caractère monétaire du registre ne dépasse jamais $10 \%$ par mois ( $c f$. tableau II et graphique 1). Il semble donc que la nouvelle valeur du gros ait eu du mal à entrer dans les faits et que les autorités n'aient pas véritablement cherché à imposer immédiatement leur décision.

Dès lors, on peut considérer que la décision royale du 12 avril 1421 n'avait pas véritablement modifié le comportement des Normands qui continuaient à exprimer les prix en monnaie de compte, comme si de rien n'était, en rappelant toutefois presque systématiquement que les gros utilisés avaient dorénavant un cours légal fixé à 5 deniers, ou bien, au moins dans un acte sur trois, en ne mentionnant rien de la manière dont la transaction s'est effectuée.

La première dépréciation du gros, on l'a dit plus haut, n'avait pas suffi à faire cesser définitivement sa perte en valeur réelle et il fallut procéder, le premier décembre suivant, à une seconde dépréciation, presque aussi draconienne que la précédente, puisque la valeur du gros fut cette fois divisée par deux. Après cette nouvelle décision royale, l'attitude des utilisateurs du tabellionage se modifia désormais de manière beaucoup plus sensible.

${ }^{24}$ Fol. 13. De même, un bourgeois rouennais qui venait d'acheter une rente à vie à un habitant de Rosay se contentait, pour sa part, d'indiquer que celle-ci se paierait en telle monnoye comme le roy se paiera en son domaine, tandis qu'un autre bourgeois qui revendait une rente en blé acceptait d'être payé en plusieurs fois en telle monnoye et pour tel pris qu'il courra au jour desdis paiemens. Fol. 253 bisv et fol. 255. Même chose encore le 6 décembre, Fol. 260, deux fois le 13 décembre, Fol. 262 et le 14 décembre Fol. 263v.

25 Fol. 108. 
Tableau II: Types de transactions entre le 12 avril et le 30 novembre 1421

\begin{tabular}{|c|c|c|c|c|c|c|}
\hline & $\begin{array}{c}\text { Nombre } \\
\text { total } \\
\text { d'actes }\end{array}$ & $\begin{array}{c}\text { en faible } \\
\text { monnaie } \\
\text { (12 gros } \\
\text { par livre) }\end{array}$ & $\begin{array}{c}\text { en forte } \\
\text { monnaie } \\
\text { (48 gros } \\
\text { par livre) }\end{array}$ & en or & $\begin{array}{c}\text { autre } \\
\text { monnaie }\end{array}$ & $\begin{array}{c}\text { aucune } \\
\text { mention } \\
\text { de } \\
\text { monnaie }\end{array}$ \\
\hline avril & 95 & $44(\mathbf{4 6 , 3 \%})$ & $6(6,3 \%)$ & $7(6,4 \%)$ & $2(\mathbf{2 , 1 \%})$ & $37(38,9 \%)$ \\
\hline mai & 218 & $52(\mathbf{2 3 , 9 \%})$ & $3(1,4 \%)$ & $11(\mathbf{5 , 2 \%})$ & $2(\mathbf{0 , 9 \%})$ & $150(\mathbf{6 8 , 8 \%})$ \\
\hline juin & 151 & $86(\mathbf{5 6 , 9 \%})$ & $3(2 \%)$ & $11(7,3 \%)$ & $1(0,7 \%)$ & $50(\mathbf{3 3 , 1 \%})$ \\
\hline juillet & 72 & $33(\mathbf{4 5 , 8 \%})$ & $0(\mathbf{0} \%)$ & $12(\mathbf{1 6}, \mathbf{7 \%})$ & $3(4,2 \%)$ & $24(33,3 \%)$ \\
\hline août & 46 & $25(54,3 \%)$ & $2(4,4 \%)$ & $2(\mathbf{4 , 4 \%})$ & $0(\mathbf{0 \%})$ & $17(\mathbf{3 6 , 9 \%})$ \\
\hline septembre & 71 & $33(\mathbf{4 6 , 5 \% )}$ & $5(7 \%)$ & $11(\mathbf{1 5}, \mathbf{5} \%)$ & $1(1,4 \%)$ & $21(\mathbf{2 9}, \mathbf{6} \%)$ \\
\hline octobre & 79 & $26(32,9 \%)$ & $7(\mathbf{8 , 9 \%})$ & $14(\mathbf{1 7}, \mathbf{7 \%})$ & $0(\mathbf{0 \%})$ & $32(\mathbf{4 0 , 5 \%})$ \\
\hline novembre & 92 & $33(\mathbf{3 5 , 9 \%})$ & $8(\mathbf{8 , 7 \%})$ & $17(\mathbf{1 8 , 5} \%)$ & $0(\mathbf{0 \%})$ & $34(36,9 \%)$ \\
\hline
\end{tabular}

N.B.: Le nombre entre parenthèses représente le pourcentage par rapport au nombre total d'actes.

Graphique 1: Types de transactions (12 avril-30 novembre 1421)

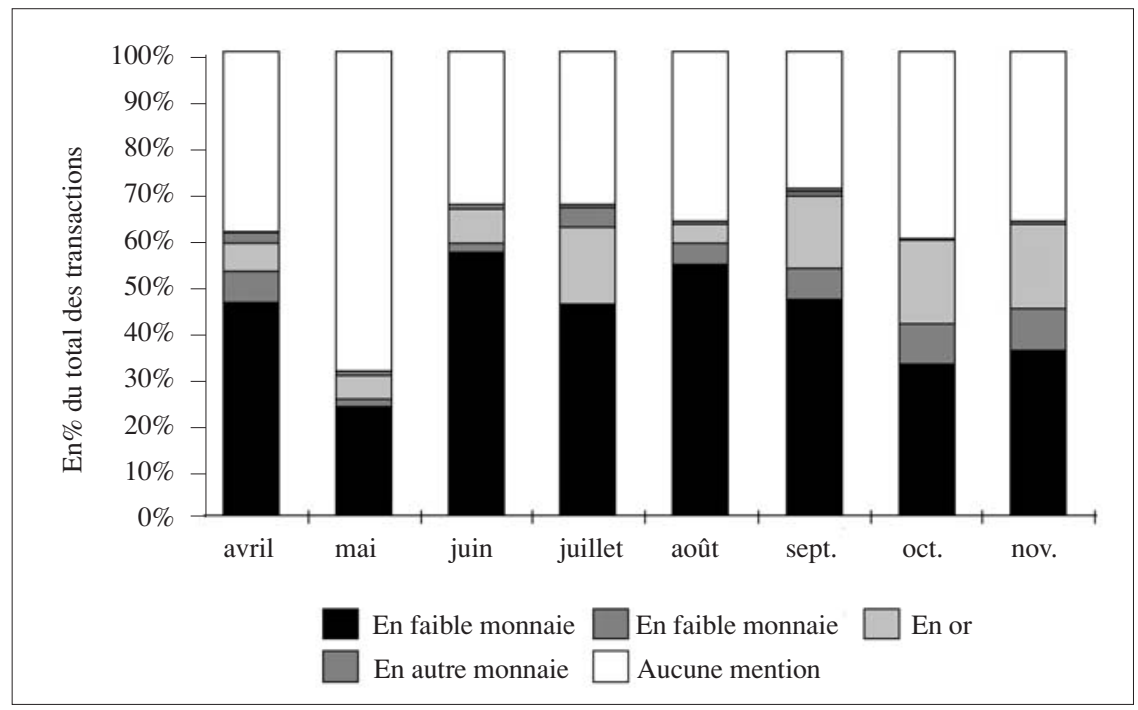

D'abord, le nombre des actes enregistrés par le tabellion redevint plus important, notamment au cours de l'hiver 1421-1422, comme si l'activité économique connaissait une relative reprise. En décembre 1421 et en janvier 1422, le nombre d'actes n'indiquant rien de la manière dont la transaction avait été effectuée tomba en dessous de $20 \%$ ( $c f$. tableau III et graphique 2). Il s'agissait dans la plupart des 
Tableau III: Types de transactions entre le $1^{\text {er }}$ décembre 1421 et le 26 septembre 1422

\begin{tabular}{|c|c|c|c|c|c|c|}
\hline & $\begin{array}{c}\text { Nombre } \\
\text { total } \\
\text { d'actes }\end{array}$ & $\begin{array}{c}\text { en faible } \\
\text { monnaie } \\
(12 \text { gros } \\
\text { par livre) }\end{array}$ & $\begin{array}{l}\text { en bonne } \\
\text { et forte } \\
\text { monnaie } \\
\text { (dont } x \\
\text { doubles } \\
\text { nouveaux) }\end{array}$ & en or & $\begin{array}{l}\text { en autre } \\
\text { monnaie }\end{array}$ & $\begin{array}{c}\text { aucune } \\
\text { mention } \\
\text { de } \\
\text { monnaie }\end{array}$ \\
\hline $\begin{array}{l}\text { décembre } \\
1421\end{array}$ & 150 & $\begin{array}{c}75 \\
(\mathbf{5 0 \%})\end{array}$ & $\begin{array}{c}15 \\
(\mathbf{1 0 \%})\end{array}$ & $\begin{array}{c}28 \\
(\mathbf{1 8 , 7 \%})\end{array}$ & $\begin{array}{c}3 \\
(2 \%)\end{array}$ & $\begin{array}{c}29 \\
(\mathbf{1 9 , 3 \%})\end{array}$ \\
\hline $\begin{array}{l}\text { janvier } \\
1422\end{array}$ & 163 & $\begin{array}{c}70 \\
(42,9 \%)\end{array}$ & $\begin{array}{c}12 \text { dont } \\
2(7,4 \%)\end{array}$ & $\begin{array}{c}46 \\
(\mathbf{2 8 , 2 \%})\end{array}$ & $\begin{array}{c}3 \\
(1,9 \%)\end{array}$ & $\begin{array}{c}32 \\
(\mathbf{1 9 , 6 \%})\end{array}$ \\
\hline février & 187 & $\begin{array}{c}77 \\
(41,2 \%)\end{array}$ & $\begin{array}{c}32 \text { dont } 3 \\
(\mathbf{1 7 , 1 \%})\end{array}$ & $\begin{array}{c}26 \\
(\mathbf{1 3 , 9 \%})\end{array}$ & $\begin{array}{c}7 \\
(3,7 \%)\end{array}$ & $\begin{array}{c}45 \\
(\mathbf{2 4 , 1 \%})\end{array}$ \\
\hline mars & 236 & $\begin{array}{c}87 \\
(36,9 \%)\end{array}$ & $\begin{array}{c}29 \text { dont } 5 \\
(\mathbf{1 2 , 3 \% )}\end{array}$ & $\begin{array}{c}51 \\
(\mathbf{2 1}, \mathbf{6} \%)\end{array}$ & $\begin{array}{c}2 \\
(\mathbf{0 , 8} \%)\end{array}$ & $\begin{array}{c}67 \\
(28,4 \%)\end{array}$ \\
\hline avril & 119 & $\begin{array}{c}27 \\
(22,7 \%)\end{array}$ & $\begin{array}{c}26 \text { dont } 4 \\
(\mathbf{2 1 , 8 \% )}\end{array}$ & $\begin{array}{c}27 \\
(22,7 \%)\end{array}$ & $\begin{array}{c}1 \\
(\mathbf{0 , 8 \%})\end{array}$ & $\begin{array}{c}38 \\
(\mathbf{3 1}, 9 \%)\end{array}$ \\
\hline mai & 113 & $\begin{array}{c}4 \\
(3,5 \%)\end{array}$ & $\begin{array}{c}20 \text { dont } 8 \\
(17,7 \%)\end{array}$ & $\begin{array}{c}39 \\
(\mathbf{3 4 , 6 \%})\end{array}$ & $\begin{array}{c}4 \\
(3,5 \%)\end{array}$ & $\begin{array}{c}46 \\
(\mathbf{4 0 , 7 \%})\end{array}$ \\
\hline juin & 90 & $\begin{array}{c}0 \\
(\mathbf{0 \%})\end{array}$ & $\begin{array}{c}28 \text { dont } 2 \\
(\mathbf{3 1 , 1 \%})\end{array}$ & $\begin{array}{c}22 \\
(\mathbf{2 4}, \mathbf{4} \%)\end{array}$ & $\begin{array}{c}1 \\
(\mathbf{1}, \mathbf{1} \%)\end{array}$ & $\begin{array}{c}39 \\
(\mathbf{4 3}, \mathbf{4 \%})\end{array}$ \\
\hline juillet & 62 & $\begin{array}{c}0 \\
(\mathbf{0 \%})\end{array}$ & $\begin{array}{l}13 \text { dont } 2 \\
(\mathbf{2 0 , 9 \%})\end{array}$ & $\begin{array}{c}10 \\
(\mathbf{1 6 , 2 \%})\end{array}$ & $\begin{array}{c}0 \\
0 \%)\end{array}$ & $\begin{array}{c}39 \\
(62,9 \%)\end{array}$ \\
\hline août & 74 & $\begin{array}{c}0 \\
(\mathbf{0 \%})\end{array}$ & $\begin{array}{l}15 \text { dont } 8 \\
(\mathbf{2 0 , 2 \%})\end{array}$ & $\begin{array}{c}16 \\
(\mathbf{2 1}, \mathbf{6} \%)\end{array}$ & $\begin{array}{c}2 \\
(2,7 \%)\end{array}$ & $\begin{array}{c}41 \\
(\mathbf{5 5}, \mathbf{5} \%)\end{array}$ \\
\hline septembre & 47 & $\begin{array}{c}0 \\
(\mathbf{0 \%})\end{array}$ & $\begin{array}{l}10 \text { dont } 2 \\
(\mathbf{2 1 , 3 \% )}\end{array}$ & $\begin{array}{c}9 \\
(\mathbf{1 9}, \mathbf{1 \%})\end{array}$ & $\begin{array}{c}1 \\
(2,1 \%)\end{array}$ & $\begin{array}{c}27 \\
(\mathbf{5 7 , 5 \%})\end{array}$ \\
\hline
\end{tabular}

N.B.: Les actes mentionnant des paiements en or peuvent faire intervenir plusieurs types de pièces en même temps.

cas de ventes et de reventes de rentes, ou d'actes qui faisaient intervenir des représentants du roi ou de la ville de Rouen. Les fermes des aides, en particulier, ne faisaient l'objet d'aucune mention de monnaie, comme si le fait qu'on utilisât la monnaie de compte criée le $1^{\text {er }}$ décembre précédent allait de soi. Dans tous les autres cas, il apparaissait nécessaire aux clients du tabellion de bien indiquer la manière dont avaient été comptées les sommes mises en jeu, ce qui était évidemment devenu indispensable étant donné les mutations de monnaie qui venaient d'avoir lieu.

Les actes qui ne tiennent apparemment pas compte de la nouvelle décision royale, établis par conséquent en faible monnaie, représentent pourtant encore presque la moitié du total jusqu'à la fin du mois de janvier et plus d'un tiers du total jusqu'en mars 1422. Au cours de cette période, les actes indiquent en général que la transaction a été effectuée en gros de 20 deniers et précise qu'il 
Graphique 2: Types de transactions ( $1^{\mathrm{er}}$ décembre 1421-septembre 1422)

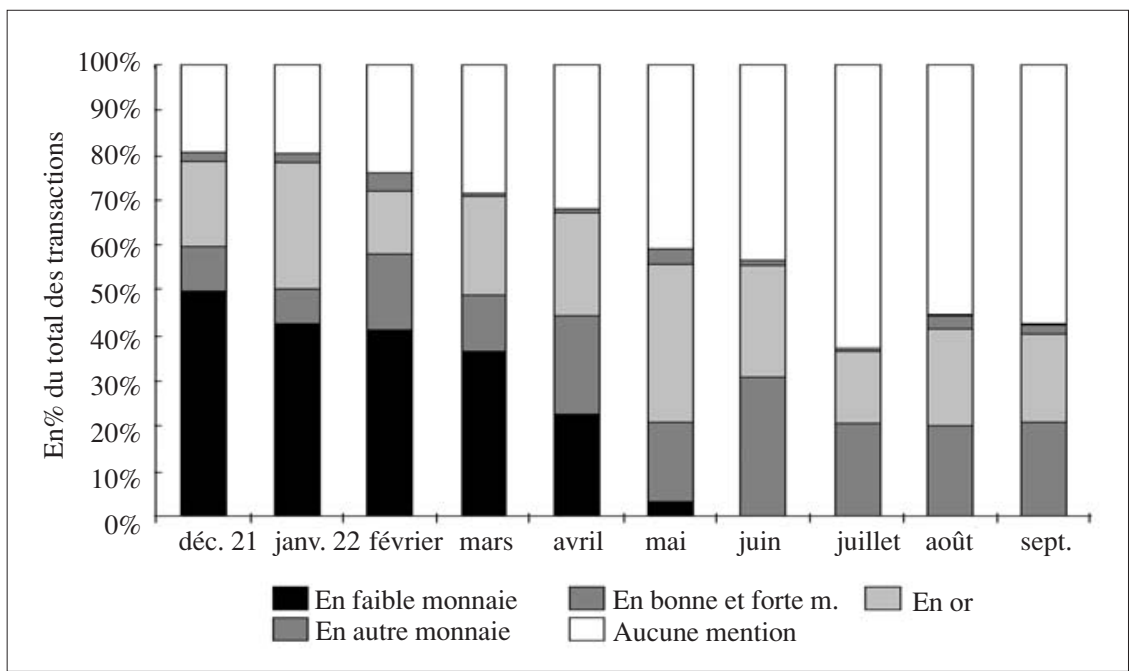

s'agit de ces pièces qui valaient 20 deniers avant Pâques 1421, qui ont ensuite valu 5 deniers jusqu'au $1^{\text {er }}$ décembre et qui ne valent plus que 2 deniers et demi à la date de la transaction. Parallèlement, au cours de cette période, les actes exprimés en forte monnaie, c'est-à-dire indiquant que l'on compte désormais 96 gros pour une livre, deviennent progressivement plus nombreux et représentent une transaction sur 4 ou sur 5, même si on trouve encore quelques actes qui choisissent comme monnaie de compte pour la transaction qu'ils enregistrent, la valeur de 48 gros par livre ${ }^{26}$.

C'est à partir du mois de mars 1422 que les choses changent assez nettement. Les actes en faible monnaie deviennent de moins en moins nombreux jusqu'à finalement disparaître complètement au cours du mois de mai. A cette date, en effet, le gros avait été décrié et les pièces mises au billon ${ }^{27}$. On constate toutefois que les usagers du tabellion avaient anticipé cette décision et avaient progressivement abandonné les gros théoriquement de 20 deniers, ce qui s'explique essentiellement par la mise en circulation des doubles deniers qui pouvaient se substituer au gros. Les transactions en forte monnaie, c'est-à-dire le plus souvent en doubles deniers ou comptées à 96 gros par livre deviennent plus fréquentes qu'avant et se fixent autour de $20 \%$ du total. A partir du mois de mai, on indique que les transactions se sont effectuées en bonne et forte monnoye, ce qui signifie

${ }^{26}$ Fol. 255. Encore le 11 février suivant, un paiement de 20 livres entre marchands se fait en comptant 48 gros pour livre. Fol. 319.

${ }^{27}$ Des lettres de Charles VI de juin 1422 décriaient toute monnaie blanche d'une valeur supérieure à 2 deniers. Il ne restait donc plus que les doubles nouveaux et des pièces de valeur inférieure. Ordonnances des rois de France, T.XI, p.163-164. Il est clair que la même décision a été prise par les autorités anglaises en Normandie. 
que l'on a utilisé les nouvelles pièces qui ont été mises en circulation, c'est-à-dire les doubles nouveaux et éventuellement même des pièces d'or.

En effet, et ce n'est pas le moins important, les actes qui ne disent rien du système de comptabilité qui a été employé représentent une proportion régulièrement croissante. On en compte un tiers du total en avril 1421 et le double à partir de juillet. Dès lors, environ plus d'un acte sur deux est enregistré sans mention de la monnaie utilisée. A partir de juin 1422, la formule, en monnoye courant à présent, autrement dit, sans plus de précision, en bonne et forte monnaie, est devenue la plus fréquente ${ }^{28}$. On peut donc considérer que la crise monétaire mais pas la hausse des prix - n'a été véritablement ressentie par les utilisateurs normands du tabellionage que de décembre 1420 à mars 1422 et qu'elle est virtuellement terminée à cette date puisqu'une confiance certaine est revenue dans les transactions ${ }^{29}$.

Ce qui est donc remarquable tient au fait qu'il s'est mis en place un double système de comptabilité utilisant soit de la «forte monnaie», c'est-à-dire tenant compte immédiatement des décisions royales, soit, le plus fréquemment, de la «monnaie faible», continuant à accorder au gros une valeur théorique de 20 deniers. Il y eut donc pendant un certain temps une situation particulièrement confuse qui ne donnait pas forcément lieu à des mentions précises et qui, par conséquent, ne peut que gêner l'historien qui néglige d'en tenir compte.

L'idée la plus souvent admise à propos de la crise monétaire du début des années 1420 est qu'elle a abouti à une très forte hausse des prix. Les chroniqueurs, par exemple le Bourgeois de Paris ou le Rouennais Pierre Cochon, se plaignent à intervalles réguliers de la hausse vertigineuse d'un certain nombre de prix, particulièrement de ceux des produits alimentaires ou de certains autres produits de première nécessité comme le bois. C'est également l'impression que donnent certaines comptabilités et on peut en trouver la confirmation dans un acte du tabellionage du 29 mars 1422. Pour justifier la demande de diminution de la rente qu'il devait à son propriétaire pour l'exploitation de trois pièces de terre, un paysan se plaignait en effet de la chierté du temps. Malheureusement, c'est la seule mention de ce type pour l'ensemble du registre et les autres demandes d'aménagement de rentes ou de baux évoquent les guerres qui ont couru et courent encore en ce pais de Normendie. Le paysan dont nous venons de parler ne faisait d'ailleurs intervenir la hausse des prix qu'après avoir expliqué qu'il n'avait pas pu labourer les terres qu'il exploitait à l'occasion et par le fait et empeschement de la guerre ${ }^{30}$. De même, le Bourgeois de Paris note que certains métaux comme le cuivre ou l'étain continuaient à être vendus à des prix qui n'avaient pas connu d'augmentation ${ }^{31}$. Certes, il est probable que cette remarque est destinée à indiquer que les métaux vils qui entraient dans la composition des pièces noires étaient si abondants que

${ }^{28}$ La première mention est du 7 juin. Fol. 422.

29 On peut sans doute voir une manifestation du rétablissement de la situation monétaire dans le fait que le roi Henry VI - autrement dit le régent Bedford - ait décidé le 23 novembre 1422 de frapper à nouveau des blancs de 10 deniers. Arch. Nat. de France, Z 1b 58, fol. 172.

30 Fol. 372.

31 Journal du Bourgeois de Paris, 1990, pp. 138-139, (242), pour l'année 1419. 
leur prix ne pouvait pas augmenter. Il n'en demeure pas moins qu'il constate que la hausse n'est pas aussi généralisée qu'il l'affirme par ailleurs.

La division par quatre, puis par huit, de la valeur légale du gros posait à ceux qui étaient liés par des contrats à caractère financier le problème crucial de savoir comment il fallait désormais transposer en monnaie de compte les sommes précédemment indiquées en gros courant théoriquement pour 20 deniers. C'est pourquoi les mentions de faible ou de forte monnaie dans les actes signifient qu'il était possible de calculer les sommes mises en jeu de manières diverses et qu'il y avait dans les faits, jusqu'à la démonétisation du gros, au moins deux échelles qui permettaient d'exprimer les prix en monnaie de compte.

Le meilleur exemple de cette situation se trouve enregistré à la date du 17 décembre 1421. Le montant du transport de 9 queues et demie de vin et d'une quantité indéterminée de baterie d'airain de Rouen à Londres est fixé à 1060 livres tournois de faible monnaie à 12 gros pour livre ce qui, est-il indiqué dans l'acte, équivaut à 132 livres 10 sous et un denier en forte monnaie (soit 8 fois moins) et, en écus d'or, à 117 écus, 17 sous et un denier obole. Ainsi, la même somme pouvait sembler 8 fois plus élevée selon qu'elle était exprimée en faible ou en forte monnaie, alors qu'elle représentait la même valeur monétaire. Dans un cas, on n'avait pas tenu compte de la décision royale et on avait compté en «anciens gros », dans l'autre on avait appliqué la réforme et donc diminué le montant en monnaie de compte de la somme à enregistrer en le divisant par $8^{32}$. Un autre exemple montrant le même phénomène se trouve enregistré à la date du 17 février suivant. L'acte mentionne, en effet, que la vente de 25 mines de froment à un Rouennais s'élevait à 16 livres de bonne monnaie, ce qui équivalait à 128 livres (soit exactement 8 fois plus) au pois de fieble monnoye à 12 gros pour livre naguère ayans cours ${ }^{33}$.

Le montant de la transaction du 17 décembre que l'on vient de mentionner était également estimé en écus. Ceux-ci avaient, à cette date, un cours officiel de 22 sous 6 deniers, si on exprimait cette valeur en forte monnaie. Par contre, bien sûr, si on continuait à compter en faible monnaie, c'est-à-dire avec un gros valant 8 fois moins que sa valeur officielle, on obtenait un écu d'or à 9 livres, ce qui correspond aux chiffres que l'on peut rencontrer ailleurs dans ce registre. D'autres exemples confirment en effet cette valeur variable des pièces d'or selon la monnaie de compte dans laquelle elle était exprimée. Ainsi, le 15 janvier 1422, une rente à vie de 100 sous est vendue pour 45 livres de faible monnaie et le tabellion indique que le vendeur confessa avoit receu ladicte somme en quatre escus d'or pour 36 livres et le demourant en gros ${ }^{34}$. Ainsi, le montant de l'écu d'or semblait

${ }^{32}$ Fol. 266v. Notons qu'il s'agit d'un acte qui fait intervenir le chambellan du duc de Bedford, autrement dit quelqu'un qui devrait théoriquement tenir compte des décisions royales. Cela montre, par conséquent, la nécessité de préciser les choses clairement pour qu'il n'y ait aucune équivoque ensuite.

${ }^{33}$ Fol. 313v. Le compte de l'Ascension 1421 du château de Domfront indique que le vicomte qui tient les registres doit à cette date 2264 livres 13 sous 8 deniers obole en gros à 5 deniers et 1164 livres 13 sous 9 deniers en gros à 20 deniers qui valent en gros à cinq deniers 291 livres 3 sous 5 deniers poitevine (c'est-à-dire quatre fois moins). Le déficit total du compte se situant donc à 2565 livres 1 denier obole en forte monnaie. Cormier, (1989), p. 704.

${ }^{34}$ Fol. 293. 
s'établir à 9 livres, mais comme l'ensemble de la transaction était réalisé en faible monnaie, autrement dit en gros de 20 deniers dont la valeur avait été divisée par 8 , on obtient un écu qui vaut bien 22 sous 6 deniers de forte monnaie, comme l'avaient établi les différentes ordonnances royales. De même, le 9 mars 1422, une reconnaissance de dettes entre des marchands de Bayeux et de Mantes enregistrée pour 106 livres de faible monnaie, à payer en icelle monnoye de gros ou en nobles ou escus, précisait que chaque noble valait 18 livres d'icelle monnoye, c'est-à-dire de faible monnaie, soit 8 fois la valeur officielle du noble $(45 \times 8=360$ sous $=18$ livres) $)^{35}$. Encore le 4 avril, pour la vente d'une pièce de vigne, les deux parties se mirent d'accord sur un montant fixé à 18 écus d'or que le vendeur confessa avoir eulz et recheus en gros à 9 douzaines pour chacun escu ${ }^{36}$.

Ce double mode de calcul concernait toutes les pièces d'or en circulation. Le 24 décembre 1421, la vente d'une maison située dans la paroisse de Saint-Maclou de Rouen, se faisait au prix de 40 livres payées en 6 moutons d'or et le reste en gros. Autrement dit, on peut admettre que, dans cette transaction, le mouton d'or avait été évalué à 6 livres soit 8 fois sa valeur en forte monnaie $(15$ x $8=120$ sous, soit 6 livres). La transaction avait donc été effectuée en 6 moutons soit 36 livres et 4 livres en gros, soit 384 pièces qui, comme le rappelle le texte de l'acte, valaient 20 deniers avant Pâques puis avaient été ramenées à 5 deniers et enfin à 2 deniers et demi. Un autre exemple, encore plus clair, daté du 12 janvier amène à la même conclusion. Deux habitants de Bailleul qui avaient acheté un certain nombre de bovins à un Rouennais pour 138 livres en faible monnaie, effectuèrent leur transaction en couronnes d'or dont la valeur de compte était fixée à 9 livres, autrement dit à 8 fois la valeur de cette pièce en forte monnaie qui était la même que celle de l'écu d'or, soit 22 sous 6 deniers ${ }^{37}$.

Le problème du choix de la manière d'exprimer une somme après les deux dépréciations du gros était donc crucial parce qu'il pouvait aboutir à des abus ou des distorsions, volontaires ou non ${ }^{38}$. En avril 1422, un débirentier qui devait payer 21 sous de rente demanda à son crédirentier d'obtenir une remise de la somme qu'il lui devait parce que, selon lui, la maison sur laquelle pesait la rente, estoit trop chargiée. Il demandait donc à son crédirentier qu'il voulsist faire rabes ou relache ou au moins prendre les deniers du pris du roy nostre sire. Cela signifie par conséquent que, plus d'un an après la première dépréciation, il y avait encore des flottements et on ne savait pas toujours s'il fallait compter les rentes en

${ }^{35}$ Rappelons qu'un noble valait 45 sous. Si on fait le calcul, on obtient 45 x $8=360$ sous, soit 18 livres. Fol. 349.

${ }^{36}$ Fol. 376v. Ainsi, si l'on considère comme avant la dépréciation du gros qu'il y en a douze par livre, cela signifie que l'écu vaut 9 livres et si l'on se place après la deuxième dépréciation, au moment où le gros vaut deux deniers et demi, on obtient : 9 × 12 × 2,5 = 270 deniers, soit 22 sous 6 deniers, ce qui est, on le sait, le cours officiel de l'écu.

${ }^{37}$ Fol. 289. De même, le rachat de rente dont nous avons parlé plus haut (note 19) qui fixait la valeur de l'écu à 7 livres le 26 septembre 1421 correspondait en fait à la valeur en faible monnaie du vieil écu d'or valant 35 sous puisque, à cette date, la valeur du gros n'avait été divisée que par 4 ( 7 livres $=140$ sous $; 140$ sous : $4=35$ sous).

38 On retrouve donc ici l'idée selon laquelle la monnaie de compte n'a pas une valeur absolue, mais est toujours liée à une monnaie réelle qui avait été mise en lumière par Van Werveke, 1934, p. 123-152. Voir aussi Depeyrot, 1995, p. 245 et p. 333. 
faible ou en forte monnaie. Il est évident que si le crédirentier continuait à exiger les 21 sous de rente en monnaie de compte, comme si aucune dévaluation n'avait eu lieu, il multipliait par 8 son revenu, et que, inversement, si le débirentier obtenait que la rente fût comptée en forte monnaie, il ne payait plus que le $1 / 8$ de la somme qui lui était réclamée ${ }^{39}$. C'est à cette même difficulté que s'était heurté le fermier de la prévôté de Neufchâtel-en-Bray qui avait demandé, le 26 février 1422, une diminution de sa ferme dont le montant était exprimé en gros. Du fait de la diminution de la valeur de cette pièce, il n'était plus en mesure de payer ce qu'il devait parce que, comme il l'expliquait, il percevait des taxes pesant sur les marchandises qui passaient par la ville, en venant de Picardie et en allant vers Rouen et la Bretagne, en pièces de faible valeur et il craignait de devoir payer 4 ou 8 fois le montant de sa ferme ${ }^{40}$.

Le fait de préciser, pour chaque transaction, la manière dont elle avait été réalisée, avait pour autre intérêt d'éviter des problèmes lors des rachats de rente ou de ventes à héritage. A plusieurs reprises, en effet, les actes contiennent des formules indiquant que les rachats devaient se faire selon la manière qui avait été enregistrée ou en semblable monnoye ${ }^{41}$. Au mois d'août 1422, à un moment où la crise pouvait être considérée comme provisoirement terminée, la vente d'une rente à vie entre deux nobles prévoyait que le raquet pouvait se faire dans les deux ans en utilisant éventuellement autre essence de monnoye, l'acheteur ne pouvant fère ne porter aucun procès ${ }^{42}$. Cette pratique était relativement courante et donnait lieu à des accords qui ne semblent pas avoir posé de problèmes. Ainsi, le 4 septembre 1422 , une veuve qui avait fait don, après la première dépréciation du gros, de ses biens évalués à 60 livres en faible monnaie, contre la prise en charge de ses besoins par le bénéficiaire du don, estimant que la solution qu'elle avait choisie ne lui convenait plus, récupéra l'ensemble de ses biens contre la somme de 22 livres et demie en monnoye courant de présent, soit seulement 45 livres de la faible monnaie qui avait cours au moment de l'acte, parce que l'ex-donateur se faisait aussi payer l'entretien de cette veuve pendant près de six mois ${ }^{43}$.

De ce qui précède découle une conclusion qui doit être prise en compte lorsque l'on veut faire des études de prix au moment des crises monétaires : des transactions, et a fortiori des prix, ne peuvent être comparés que s'ils sont exprimés dans la même monnaie de compte. C'est à partir de cette précaution seulement qu'une étude des prix peut être véritablement parlante. C'est d'ailleurs ce que montre le Bourgeois de Paris. Un certain nombre de conflits éclatèrent dans la capitale,

${ }^{39}$ Fol. 393v. Le débirentier réussit à obtenir une baisse de 11 sous sur les 21 qu'il devait en donnant 4 écus d'or au crédirentier, ce qui ne correspondait pas totalement à la dévaluation du gros. C'est aussi le risque de voir les rentes et les loyers multipliés par 8 que déplore le Journal du Bourgeois de Paris, 1990, p. 173-174 (321).

${ }^{40}$ Bibl. Nat. de France, Ms français, 26044, [5705]. Il obtient une division par quatre de sa ferme, à compter entre le 12 avril 1421 et le ler décembre 1421.

${ }^{41}$ Plus précis encore, un acte du 17 octobre 1421, enregistrant la reconnaissance de dette d'un prisonnier à l'Anglais qui l'avait capturé, évaluée à 12 écus d'or, était a paier en ladicte monnoye d'or ou en monnoye qui vaille iceulx escus. Fol. 213v.

${ }^{42}$ Fol. 445. La rente fut d'ailleurs rachetée pratiquement deux ans plus tard, le 9 août 1424, apparemment sans difficulté.

43 Fol. 461. 
parce qu'on ne savait pas comment on devait désormais calculer les prix ${ }^{44}$. De même, lorsque Pierre Cochon indiquait que l'écu valait 8 à 9 livres, il ne faisait que constater une situation en faible monnaie ${ }^{45}$. Il est d'ailleurs probable que le maintien de l'utilisation fréquente de la faible monnaie de gros pendant plus de dix mois, malgré deux dépréciations, était dû au fait qu'on ne savait pas vraiment comment compter les sommes mises en jeu en tenant compte de leurs nouvelles valeurs.

Il serait évidemment nécessaire d'essayer de comparer les prix aux salaires de ceux qui avaient leur travail pour ressource essentielle. Malheureusement, pour la période de la crise monétaire, l'étude des salaires est difficile à réaliser parce que la plupart des comptabilités qui couvraient cette période ont disparu et que les données contenues dans les quittances de la Bibliothèque Nationale sont peu probantes. Heureusement, il reste le compte de la fabrique de la cathédrale de Rouen pour la période de la Saint-Michel 1420 à la Saint-Michel 1421.

Comme on le voit sur le tableau IV, l'année est divisée en 8 termes et il n'est pas possible comme pour les prix contenus dans le registre du tabellionage de suivre l'évolution des salaires jour après jour. Il s'agit bien entendu de salaires en monnaie de compte et la manière dont ils ont été payés n'est jamais mentionnée même si elle peut en général se déduire assez facilement. La première constatation est que la fin de l'année 1420, pourtant marquée par la diffusion de mauvaise monnaie blanche dont on se servait à la cathédrale pour payer les ouvriers, n'aboutit à aucune modification des salaires jusqu'à Noël. Mieux même, les responsables de la fabrique n'hésitent pas, à partir de la Saint-Martin, comme lors des autres années, à baisser les salaires des maçons et du plombier pour l'hiver parce que la durée de la journée de travail diminue, ce qui aboutit à une diminution de $20 \%$ de leurs salaires. Cette situation n'était pourtant pas tenable face à la hausse des prix qui se manifestait alors à Rouen et les charpentiers ainsi que les couvreurs de tuiles obtiennent une augmentation de $25 \%$ de leurs salaires entre la SaintMichel et la Saint-Martin ${ }^{46}$. Les manœuvres avaient déjà bénéficié de la même mesure puisque leur salaire était de 2 sous 6 deniers au cours de l'année précédente, ce qui semble indiquer que les augmentations étaient accordées d'abord à ceux qui en avaient le plus besoin. Ces augmentations s'expliquent sans difficulté par le fait que ces salaires quotidiens étaient payés en gros. Les charpentiers (sauf

${ }^{44}$ Journal du Bourgeois de Paris, 1990, pp. 173-174 (321). C'est pour clarifier les choses que le 31 octobre 1421, le roi Charles VI avait adressé au prévôt de Paris des lettres qui expliquaient comment fixer le prix des denrées selon que l'on comptait en vieille ou en nouvelle monnaie. Ainsi, les sujets du roi devaient, soit se servir de la vieille monnaie au prix fixé, soit l'amener à la Monnaie de Paris pour la fondre et forger de nouvelles pièces. De nouvelles lettres du 15 décembre 1421, donc après la deuxième dépréciation du gros, précisaient à nouveau «la manière de faire les paiements relativement à la mutation des monnaies », en indiquant qu'il fallait en fait utiliser la valeur de compte de la monnaie qui avait cours au moment du contrat Ordonnances des rois de France, t. XI, p. 134 et suiv. et pp. 146-150.

45 Cochon, 1870 , p. 343. On peut considérer que, de la même manière, les valeurs de l'écu fournies par Mollat, 1953, p. 28, à partir du livre d'un changeur, sont en faible monnaie jusqu'au mois de février 1422, puis en forte monnaie.

46 Jean le Monnier, le maître des œuvres de charpenterie, a été apparemment présent sur le chantier avant l'équipe qui est engagée ensuite. 
Tableau IV:

Salaires des ouvriers de la cathédrale en monnaie de compte (faible monnaie)

(Saint-Michel 1420-Saint-Michel 1421)

(Arch. dép. de Seine-Maritime G2486)

\begin{tabular}{|c|c|c|c|c|c|c|c|c|}
\hline $\begin{array}{l}\text { Noms } \\
\text { et professions }\end{array}$ & 1 & 2 & 3 & 4 & 5 & 6 & 7 & 8 \\
\hline \multicolumn{9}{|c|}{ Appareilleur } \\
\hline Jean Roussel & $5 s$ & $4 s 6 d$ & $6 \mathrm{~s} 8 \mathrm{~d}$ & $6 \mathrm{~s} 8 \mathrm{~d}$ & $16 \mathrm{~s} 8 \mathrm{~d}$ & $16 \mathrm{~s} 8 \mathrm{~d}$ & $16 \mathrm{~s} 8 \mathrm{~d}$ & $16 \mathrm{~s} 8 \mathrm{~d}$ \\
\hline \multicolumn{9}{|c|}{ Maçons } \\
\hline Jacques Potier & $5 s$ & $4 \mathrm{~s}$ & $5 s$ & & & & & \\
\hline Colin Salvart & $4 \mathrm{~s}$ & $4 \mathrm{~s}$ & $4 \mathrm{~s}$ & $5 \mathrm{~s}$ & $10 \mathrm{~s}$ & & & \\
\hline Pierre de Plannes & & & $5 \mathrm{~s} 10 \mathrm{~d}$ & $5 \mathrm{~s} 10 \mathrm{~d}$ & & & & \\
\hline Pierre Bense & & & $5 \mathrm{~s} 10 \mathrm{~d}$ & $5 \mathrm{~s} 10 \mathrm{~d}$ & & & & \\
\hline Jean le Roy & & & & & $15 \mathrm{~s}$ & $15 \mathrm{~s}$ & $15 \mathrm{~s}$ & $15 \mathrm{~s}$ \\
\hline Raoul Briant & & & & & & $15 \mathrm{~s}$ & $15 \mathrm{~s}$ & $15 \mathrm{~s}$ \\
\hline Maugier le Sueur & & & & & $13 \mathrm{~s} 4 \mathrm{~d}$ & $13 \mathrm{~s} 4 \mathrm{~d}$ & $13 \mathrm{~s} 4 \mathrm{~d}$ & $13 s 4 d$ \\
\hline \multicolumn{9}{|c|}{ Charpentiers } \\
\hline Jean le Monnier & $5 \mathrm{~s}$ & & & $9 \mathrm{~s} 2 \mathrm{~d}$ & $9 \mathrm{~s} 2 \mathrm{~d}$ & $15 \mathrm{~s}$ & & \\
\hline Philippe Boudart & $6 \mathrm{~s} 8 \mathrm{~d}$ & & & & & & & \\
\hline Jean Vastecuir & $6 \mathrm{~s} 8 \mathrm{~d}$ & & & & & & & \\
\hline Jean Clérembault & $6 \mathrm{~s} 8 \mathrm{~d}$ & & & $7 \mathrm{~s} 6 \mathrm{~d}$ & & & & \\
\hline Jean Fayel & $6 s 8 d$ & & & & & & & \\
\hline Jacques Parey & $6 s 8 d$ & & & $9 \mathrm{~s} 2 \mathrm{~d}$ & $9 \mathrm{~s} 2 \mathrm{~d}$ & $15 \mathrm{~s}$ & & \\
\hline Colin Maçon & & & & $9 \mathrm{~s} 2 \mathrm{~d}$ & $9 \mathrm{~s} 2 \mathrm{~d}$ & $15 \mathrm{~s}$ & & \\
\hline Pierre Lotin & & & & & $6 \mathrm{~s} 8 \mathrm{~d}$ & & & \\
\hline Jean Fagues & & & & & & $13 \mathrm{~s} 4 \mathrm{~d}$ & & \\
\hline Jean Eudes & & & & & & $13 \mathrm{~s} 4 \mathrm{~d}$ & & \\
\hline \multicolumn{9}{|c|}{ Couvreurs de tuiles } \\
\hline Thomas le Viautre & $6 s 8 d$ & $6 s 8 d$ & & & & $18 \mathrm{~s} 4 \mathrm{~d}$ & $18 \mathrm{~s} 4 \mathrm{~d}$ & \\
\hline Pierre le Viautre & $6 s 8 d$ & $6 s 8 d$ & & & & & & \\
\hline \multicolumn{9}{|l|}{ Mathieu Herpin } \\
\hline \multicolumn{9}{|c|}{ Plâtriers } \\
\hline Colin de la Haye & & $6 s 8 d$ & & & & & & \\
\hline Et. de la Boissière & & & & & & $8 s 4 d$ & & \\
\hline
\end{tabular}


Tableau IV (suite)

\begin{tabular}{|c|c|c|c|c|c|c|c|c|}
\hline $\begin{array}{l}\text { Noms } \\
\text { et professions }\end{array}$ & 1 & 2 & 3 & 4 & 5 & 6 & 7 & 8 \\
\hline \multicolumn{9}{|c|}{ Plombier } \\
\hline A. Delebecourt & & $4 \mathrm{~s}$ & & & & $15 \mathrm{~s}$ & & \\
\hline \multicolumn{9}{|c|}{ Ouvriers de bras } \\
\hline Jean Campion & $3 \mathrm{~s} 4 \mathrm{~d}$ & $3 \mathrm{~s} 4 \mathrm{~d}$ & $3 s 4 d$ & & & & & \\
\hline Robert Capendu & & $3 \mathrm{~s} 4 \mathrm{~d}$ & & & & & & \\
\hline Robert Rogier & & $3 \mathrm{~s} 4 \mathrm{~d}$ & & & & & & \\
\hline Pierre Barnot & & & & $5 \mathrm{~s}$ & & & & \\
\hline Jean Barbot & & & & $5 \mathrm{~s}$ & $6 s 8 d$ & $6 \mathrm{~s} 8 \mathrm{~d}$ & $6 \mathrm{~s} 8 \mathrm{~d}$ & $6 s 8 d$ \\
\hline Jean Burgeleu & & & & & & $6 \mathrm{~s} 8 \mathrm{~d}$ & & \\
\hline \multicolumn{9}{|c|}{ Valet de la fabrique } \\
\hline Michel Deleau & $2 \mathrm{~s} 6 \mathrm{~d}$ & $2 \mathrm{~s} 6 \mathrm{~d}$ & $2 \mathrm{~s} 6 \mathrm{~d}$ & $2 \mathrm{~s} 6 \mathrm{~d}$ & $3 \mathrm{~s} 9 \mathrm{~d}$ & $3 \mathrm{~s} 9 \mathrm{~d}$ & $3 \mathrm{~s} 9 \mathrm{~d}$ & $3 \mathrm{~s} 9 \mathrm{~d}$ \\
\hline
\end{tabular}

1 Terme de la Saint-Michel-Saint-Martin 2 Saint-Martin-Noël 3 Noël-Chandeleur (Brandons) 4 Chandeleur-Pâques 5 Pâques-Pentecôte 6 Pentecôte-Saint-Jean 7 Saint-Jean-Assomption 8 AssomptionSaint-Michel.

le maître des œuvres Jean Le Monnier) et les couvreurs qui recevaient 3 gros en reçoivent désormais 4 et les manœuvres, qui en recevaient un et demi (peut-être en blancs de 5 deniers, mais c'est peu probable), en obtiennent à présent 2. Seul, le valet de la fabrique qui bénéficiait d'une sorte d'emploi à plein temps n'a droit à aucune augmentation ${ }^{47}$. Les maçons doivent attendre Noël pour obtenir à leur tour des augmentations, mais mis à part l'appareilleur Jean Roussel, leur salaire reste inférieur à celui des charpentiers.

C'est à partir du terme de Pâques que les choses évoluent plus nettement. En effet, il faut maintenant tenir compte de la dépréciation qui a été ordonnée par le roi d'Angleterre. Le gros ne vaut plus officiellement que 5 deniers et il n'est plus question de donner ces pièces aux ouvriers de la fabrique en continuant à leur accorder une valeur de 20 deniers. Les salaires sont donc réévalués mais encore exprimés en faible monnaie car il n'était évidemment pas possible au receveur de la fabrique de donner davantage de pièces aux ouvriers et de ne pas l'indiquer dans sa comptabilité. Dans la mesure où il est amené à donner plus de gros aux ouvriers qu'avant la décision royale, il lui faut faire apparaître cette modification dans son registre établi en monnaie de compte.

Néanmoins, sans doute au terme d'un accord avec les ouvriers, il ne procède pas à une multiplication par quatre des sommes qui leur sont versées, mais seulement à une augmentation plus ou moins substantielle. Le salaire de l'appareilleur Jean Roussel n'est multiplié que par 2,5 par rapport au terme de Noël (200 deniers

${ }^{47}$ Sur ce manœuvre particulier, voir Lardin, 1996, pp. 361-373. 
Tableau V:

Salaires des ouvriers de la cathédrale en monnaie de compte (faible monnaie)

(Saint-Michel 1420-Saint-Michel 1421)

(Arch. dép. de Seine-Maritime G2486)

\begin{tabular}{|c|c|c|c|c|c|c|c|c|}
\hline $\begin{array}{l}\text { Noms } \\
\text { et professions }\end{array}$ & 1 & 2 & 3 & 4 & 5 & 6 & 7 & 8 \\
\hline \multicolumn{9}{|c|}{ Appareilleur } \\
\hline Jean Roussel & 3 & 2,5 & 4 & 4 & 10 & 10 & 10 & 10 \\
\hline \multicolumn{9}{|c|}{ Maçons } \\
\hline Jacques Potier & 3 & 2,4 & 3 & & & & & \\
\hline Colin Salvart & 2,4 & 2,4 & 2,4 & 3 & 3,5 & & & \\
\hline Pierre de Plannes & & & 3,5 & 3,5 & & & & \\
\hline Pierre Bense & & & & & & 9 & 9 & 9 \\
\hline Jean le Roy & & & & & & 9 & 9 & 9 \\
\hline Raoul Briant & & & & & 8 & 8 & 8 & 8 \\
\hline Maugier le Sueur & & & & & & & & \\
\hline \multicolumn{9}{|c|}{ Charpentiers } \\
\hline Jean le Monnier & 3 & & & 5,5 & 5,5 & 9 & & \\
\hline Philippe Boudart & 4 & & & & & & & \\
\hline Jean Vastecuir & 4 & & & & & & & \\
\hline Jean Clérembault & 4 & & & 4,5 & & & & \\
\hline Jean Fayel & 4 & & & & & & & \\
\hline Jacques Parey & 4 & & & 5,5 & 5,5 & 9 & & \\
\hline Colin Maçon & & & & 5,5 & 5,5 & 9 & & \\
\hline Pierre Lotin & & & & & 4 & & & \\
\hline Jean Fagues & & & & & & 8 & & \\
\hline Jean Eudes & & & & & & 8 & & \\
\hline \multicolumn{9}{|c|}{ Couvreurs de tuiles } \\
\hline Thomas le Viautre & 4 & 4 & & & & 11 & 11 & \\
\hline Pierre le Viautre & 4 & 4 & & & & & & \\
\hline Mathieu Herpin & & 4 & & & & & & \\
\hline \multicolumn{9}{|c|}{ Plâtriers } \\
\hline Colin de la Haye & & 4 & & & & & & \\
\hline Et. de la Boissière & & & & & & 5 & & \\
\hline
\end{tabular}


Tableau IV (suite)

\begin{tabular}{|c|c|c|c|c|c|c|c|c|}
\hline $\begin{array}{l}\text { Noms } \\
\text { et professions }\end{array}$ & 1 & 2 & 3 & 4 & 5 & 6 & 7 & 8 \\
\hline \multicolumn{9}{|c|}{ Plombier } \\
\hline A. Delebecourt & & 2,4 & & & & 9 & & \\
\hline \multicolumn{9}{|c|}{ Ouvriers de bras } \\
\hline Jean Campion & 2 & 2 & 2 & & & & & \\
\hline Robert Capendu & & 2 & & & & & & \\
\hline Robert Rogier & & 2 & & & & & & \\
\hline Pierre Barnot & & 2 & & & & & & \\
\hline Jean Barbot & & & & 3 & 4 & 4 & 4 & 4 \\
\hline Jean Burgeleu & & & & & & 4 & & \\
\hline \multicolumn{9}{|c|}{ Valet de la fabrique } \\
\hline Michel Deleau & 1,5 & 1,5 & 1,5 & 1,5 & 2,25 & 2,25 & 2,25 & 2,25 \\
\hline
\end{tabular}

1 Terme de la Saint-Michel-Saint-Martin 2 Saint-Martin-Noël 3 Noël-Chandeleur (Brandons) 4 Chandeleur-Pâques 5 Pâques-Pentecôte 6 Pentecôte-Saint-Jean 7 Saint-Jean-Assomption 8 AssomptionSaint-Michel.

contre 80 deniers) et par 3,33 par rapport au terme de la Saint-Michel. Le salaire des autres maçons a, lui, été multiplié par trois par rapport à la Saint-Michel et ils reçoivent désormais 9 gros par jour (15 sous, soit 180 deniers) contre 3 (5 sous, soit 60 deniers) au début du compte.

Les couvreurs de tuiles qui recevaient 4 gros dès la Saint-Michel en reçoivent désormais 11 (18 sous 4 deniers, soit 220 deniers), ce qui signifie que leur salaire a seulement été multiplié par 2,75. Le sort des charpentiers est un peu différent. Leur salaire passe de 4 gros au terme de la Saint-Michel, à 5 gros et demi à la Chandeleur ( 9 sous 2 deniers, soit 110 deniers), puis sans doute à la suite de leurs protestations à 9 gros (15 sous, soit 180 deniers) à partir de Pâques. Le salaire des charpentiers qualifiés a donc été multiplié par trois ${ }^{48}$. C'est la même augmentation qui est attribuée au plombier Adam Delebecourt dont le salaire d'été était de 5 sous $^{49}$.

Les ouvriers non qualifiés sont moins favorisés. Le valet du plâtrier Colin de la Haye, voit son salaire passer de 4 gros (6 sous 8 deniers, soit 80 deniers) à 5 gros ( 8 sous quatre deniers, soit 100 deniers), ce qui représente seulement une augmentation de $20 \%$. Les ouvriers de bras dont le salaire était de 2 sous 6 deniers avant la crise ont eu, on l'a dit, une première augmentation dès la Saint-Michel et

48 Jean Eude et Jean Fagues, apparemment un peu moins qualifiés, touchent seulement 8 gros (13 sous 4 deniers, soit 160 deniers).

49 Arch. dép. de Seine-Maritime, G2485. Saint-Michel 1419-Saint-Michel 1420. 
Graphique 3:

Les salaires à la cathédrale de Rouen (1420-1421)

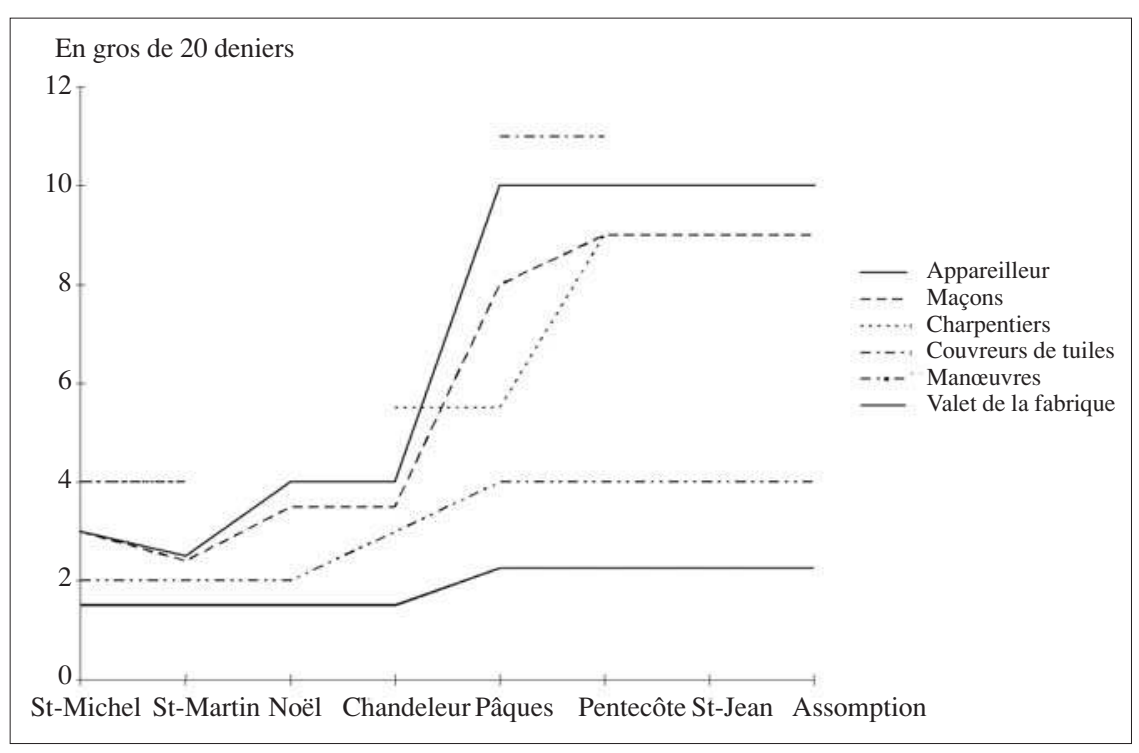

Graphique 4:

Salaires journaliers des maçons de la cathédrale de Rouen

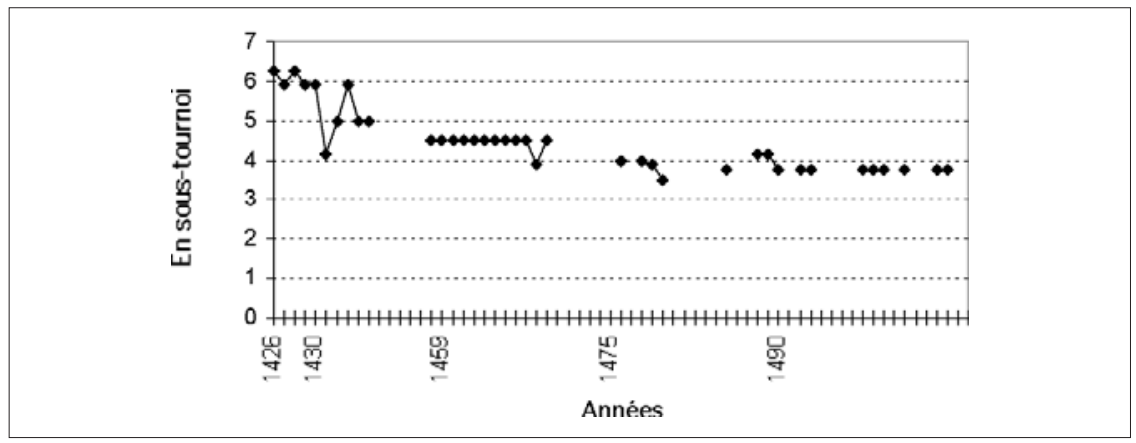

leur salaire a été fixé à 2 gros (3 sous 4 deniers, soit 40 deniers) jusqu'à la Chandeleur 1421. Après quoi, il s'est établi à 3 gros ( 5 sous, soit 60 deniers $)$ et à 4 gros (6 sous 8 deniers, soit 80 deniers) à partir de Pâques. Au total donc, par rapport à la Saint-Michel, leur salaire a été multiplié par 2 et par 2,66 si l'on se réfère à l'année précédente. Enfin, le valet de la fabrique voit son salaire passer seulement de 2 sous 6 deniers à 3 sous 9 deniers, ce qui ne représente que $50 \%$ d'augmentation.

Ainsi, on le voit, puisqu'il fallait bien tenir compte de la décision royale et considérer que les pièces utilisées avaient perdu de leur valeur, la dépréciation du 
gros a eu comme conséquence une augmentation du nombre de pièces qu'on a données aux ouvriers de la fabrique ${ }^{50}$. Pourtant, à aucun moment, on n'a procédé à une multiplication par quatre des salaires, ce qui aurait été le seul moyen de rétablir réellement la situation après le 12 avril. De plus, l'augmentation n'a pas été la même pour tous et l'appareilleur Jean Roussel s'en tire moins mal que les ouvriers de bras ou le valet de la fabrique. Dans l'ensemble, donc, on constate une fois de plus que la hausse apparente des salaires est en réalité un effet du choix du système de compte puisque si les responsables de la fabrique avaient suivi les méthodes employées par les officiels, c'est-à-dire s'ils avaient maintenu les salaires au niveau où ils étaient, mais en donnant quatre fois plus de pièces qu'avant le 12 avril aux ouvriers, ces derniers auraient finalement reçu plus d'argent qu'avec la méthode qui fut employée. C'est parce qu'ils ont continué à être comptés en gros de 20 deniers que les salaires ont apparemment augmenté. S'ils avaient été comptés en forte monnaie, c'est-à-dire en comptant 48 gros par livre, comme le montre le tableau V, non seulement ils n'auraient pas augmenté, mais même ils auraient été inférieurs à ce qu'ils étaient auparavant.

Il n'est pas possible de savoir comment les salaires de ces ouvriers évoluèrent après la seconde dévaluation puisqu'une lacune qui dure jusqu'en 1425 interrompt la série des comptes de la fabrique de la cathédrale. A cette date, on versait toujours des salaires d'hiver et d'été. Les maçons et les charpentiers touchaient 5 sous 10 deniers par jour de la Saint-Martin à Pâques et 6 sous 3 deniers en été ; les manœuvres 2 sous 1 denier en hiver et 2 sous 11 deniers en été. La situation s'était donc rétablie à des niveaux assez proches de ceux que l'on connaissait avant la crise monétaire, avec toutefois une légère hausse semblable à celle que l'on peut noter pour les prix de certains matériaux de construction. Dans le domaine des salaires, comme dans celui des prix, la crise monétaire semble, par conséquent, avoir été pratiquement jugulée dans les années 1425-1430.

La nécessité de savoir dans quel système de compte on avait effectué les calculs était un des problèmes à résoudre quand des contestations se produisaient dans les années suivantes. Au moment de la crise monétaire, le capitaine de Dieppe, dans le but de renforcer les fortifications avait obligé les représentants de la municipalité à faire des réparations à l'hôtel de la vicomté appartenant à l'archevêque de Rouen, le seigneur de la ville. Les travaux s'élevèrent à un peu plus de 600 livres-tournois. En 1424, les représentants de la ville essayèrent d'obtenir que ces dépenses leur fussent remboursées par les représentants de l'archevêque parce que, à leurs yeux, l'hôtel de la vicomté ne faisait pas partie des bâtiments dont ils avaient la charge. Ils expliquèrent, en outre, que le capitaine les avait obligés à payer ces travaux en forte monnaie alors que les travaux avaient été estimés en faible monnaie, ce qui lésait encore plus la municipalité. L'Echiquier de Rouen à qui ils avaient adressé leur réclamation refusa de l'accepter mais estima qu'il fallait néanmoins «évaluer ledit paiement à la monnaie de présent» ${ }^{51}$,

${ }^{50}$ La cathédrale ne peut pas se passer de lui à cette date car le maître des œuvres officiel, Jenson Salvart, est, à cette date, occupé sur le chantier de construction du Palais du roi Henry V, qui occupe d'ailleurs la plupart des maçons qualifiés de la ville. Brequigny, 1858, N 961, 24 février 1421. Arch. dép. de Seine-Maritime 2E1/168 Fol. 210v. 
preuve de l'incertitude dans laquelle on était quant à la valeur réelle de la somme qui était en litige.

Le problème des relations entre les monnaies réelles en circulation et la traduction de leur valeur en monnaie de compte dans les comptabilités est évidemment important lors de crises du type de celle de 1421-1422. Il est pourtant relativement aisé d'y prêter attention parce que le phénomène est connu des historiens qui en trouvent des mentions nombreuses dans les sources. La situation monétaire n'est pas toujours aussi facile à repérer et c'est parfois par hasard que l'on peut en prendre conscience. On sait qu'en 1435 la révolte du Pays de Caux réduisit la domination anglaise sur la Normandie orientale. La réaction militaire des Anglais fut vive et l'essentiel du territoire momentanément perdu fut récupéré à l'exception de la ville de Dieppe occupée par les troupes de Charles Desmarets qui ne fut rendue à son seigneur, l'archevêque de Rouen, qu'à l'occasion de la trêve de 1444. Entre temps, comme lors des régales, ce furent les officiers royaux qui administrèrent la ville et en récupérèrent les revenus au nom du roi. Il nous reste un seul compte de cette période - pour l'année 1437-1438 ${ }^{52}$ - dont les données en monnaie de compte permettent, apparemment, de connaître les évolutions des prix et de les comparer, au besoin en les déflatant, aux autres prix de la province ou du reste du royaume.

Le fait n'est malheureusement pas aussi simple qu'on pourrait le penser. En effet, lorsque la ville fut rendue à l'archevêque de Rouen, son receveur se rendit à Rouen en juillet 1447 pour y apporter sa recette. Il fut très surpris d'apprendre que les pièces d'or qu'il avait apportées avec lui n'avaient plus la même valeur à Rouen que celle qu'on leur accordait encore à Dieppe, où il n'y avait apparemment plus de changeur depuis le début du XV' siècle. Ainsi, les écus d'or que l'on comptait pour 30 sous à Dieppe valaient 9 deniers de moins à Rouen tout comme les saluts d'or dont la valeur avait beaucoup baissé depuis leur création par Henry $\mathrm{V}$ à l'occasion de la crise monétaire de $1421-1422^{53}$.

Ainsi, au moins pour la monnaie d'or - dont on ne sait jamais quand elle fut utilisée -, les données en monnaie de compte dont on peut disposer pour l'année 1437-1438 ne peuvent être utilisées qu'avec précaution puisqu'on ignore à quel moment précis s'est produite la modification de la valeur des pièces d'or circulant à Rouen et, de ce fait, l'utilisation du registre de comptabilité n'a plus qu'une valeur limitée aux domaines qu'il concerne sans que des comparaisons soient possibles avec l'extérieur de la seigneurie.

Des problèmes identiques se posent pour la fin des années 1460. Tous les auteurs ont insisté sur le fait que la période qui suivit l'occupation anglaise fut l'occasion d'une amélioration du niveau de vie des salariés, même si ce phénomène fut lent à se mettre en place et si tous les secteurs n'en tirèrent pas le même

51 ADSM $100 \mathrm{~J} 34$ [62].

52 ADSM G 505.

53 ADSM G 507 fol. 63v et 64. Le salut d'or était compté pour 30 sous 9 deniers à Dieppe et ne valait que 30 sous à Rouen. 
profit ${ }^{54}$. Un certain nombre de faits montrent, en effet, que le sort des ouvriers du bâtiment fut nettement meilleur qu'au cours des années précédentes, et également de celles qui suivirent. La rareté relative de la main-d'œuvre spécialisée amena en effet les employeurs à accorder un certain nombre d'avantages aux ouvriers : paiement de certains après-midi chômés, suppression de la diminution des salaires d'hiver, etc. Pourtant, l'étude des salaires versés par la fabrique de la cathédrale à ses ouvriers semble montrer une baisse des salaires les plus fréquemment versés ${ }^{55}$.

Cette situation ne s'explique, une fois de plus, que si l'on tient compte du lien entre les monnaies réelles en circulation et la transcription des sommes considérées dans les comptabilités. On sait, en effet, que le frère du roi Louis XI se révolta contre lui et s'empara de Rouen qui, pour quelque temps, se retrouva séparée du reste du royaume. A l'occasion de ces événements, il y eut apparemment une mutation monétaire au cours des années 1466-1468 sur laquelle on est mal renseigné. Les comptabilités enregistrent en effet à nouveau des mentions de faible et de forte monnaie qui traduisent cette mutation. Pourtant, cette fois, il ne s'agissait plus d'une dévaluation, mais apparemment d'une réévaluation. Le compte de l'archevêché de Rouen de 1466-1467 note que, pour la fonte d'une cloche, on acheta à Paris 5350 livres de métal «paié au pris de 10 frans et demy parisis forte monnoye pour chacun cent qui valent à tournois 11 livres 16 sous 3 deniers pour chacun cent ", ce qui est assez surprenant puisque 10,5 livres parisis auraient dû valoir 13 livres 2 sous 6 deniers, à moins que le franc parisis ne vaille que 0,88 livre tournois, c'est-à-dire 17 sous 6 deniers tournois ${ }^{56}$.

Les nouvelles pièces en circulation ayant une valeur plus forte que les précédentes, leur enregistrement en monnaie de compte apparaissait de prime abord comme une baisse des prix et surtout des salaires, alors que dans les faits leur valeur réelle s'était renforcée. Ainsi, une apparente baisse des salaires en monnaie de compte masquait un maintien, voire une amélioration du pouvoir d'achat des salariés ${ }^{57}$.

En conclusion, on peut donc constater que les données monétaires en monnaie de compte ne peuvent pas être séparées des réalités monétaires qu'elles enregistrent. Dans bien des cas, tel salaire ou tel prix est exprimé dans les faits en un certain nombre de pièces dont la valeur est fixée et connue de tous. Ainsi, des salaires de 2 sous 1 denier ou de 2 sous 11 deniers par jour ne semblent étonnants que lorsque l'on ne tient pas compte du fait qu'ils étaient payés en blancs de 5 deniers et correspondaient à 5 ou 7 pièces. Dans le cas des paiements plus importants, c'était la valeur des pièces d'or, comme on l'a vu à Dieppe à la fin de la Guerre de Cent ans, qui déterminait les modes de paiement et éventuellement les prix. Il est donc temps que les historiens qui s'intéressent aux phénomènes économiques

${ }^{54}$ Bois, 1976 ; Mollat, 1953 ; Lardin, 1999, pp. 141-173.

55 Ces observations contredisent celles de Guy Bois, mais il faut tenir compte du fait qu'il n'a pratiquement pas utilisé cette source.

56 A cette date, l'écu d'or valait 31sous 3 deniers tournois. ADSM G64 fol. 23-v.

${ }^{57}$ L'importance des mutations monétaires a été, notamment, mise en évidence par Menjot, 1994, pp. 222-226. 
essaient systématiquement de lier les données abstraites des comptabilités aux faits réels de la circulation monétaire, notamment en mettant en place une recension de leur utilisation à partir des données textuelles disponibles dont l'importance est sans commune mesure avec les données anecdotiques fournies par les trésors.

\section{BIBLIOGRAPHIE}

Allmand, Christopher T., Lancastrian Normandy, The History of a Medieval Occupation. 1415-1450, Oxford, 1983, trad. française Paris, 1987.

Belaubre, Jean, Histoire numismatique et monétaire de la France médiévale, Paris, le léopard d'or, 1986.

Belaubre, Jean, Les collections monétaires, vol. II, 2, Les monnaies médiévales, l'ère du gros, l'administration des monnaies, Paris, administration des monnaies et médailles, 1988.

Bois, Guy, Crise du féodalisme, Paris, FNSP, 1976, rééd. 1981.

Bréquigny, Louis G. (éd.), «Rôles normands et français et autres pièces tirées des archives de Londres par Bréquigny », 24 février 1421, éd. L. Puiseux, Mémoires de la Société des Antiquaires de Normandie, $3^{\mathrm{e}}$ série, Caen, 1858, $\mathrm{N}^{\circ} 961$.

Clémencet, Suzanne, «Z1b. Cours des monnaies», dans M. ANTOINE et alii, Guide des recherches dans le fonds judiciaire de l' Ancien Régime, Paris, 1958.

Cochon, Pierre, Chronique normande, Rouen, éd. Chrétiens. De Beaurepaire, 1870.

Cormier, Jean-Philippe, «Variations du cours de la florette en Normandie au début de l'occupation anglaise (1419-1422), d'après un compte inédit», Bulletin de la Société française de Numismatique, 1989, p. 703-705.

Day, John, Monnaies et marchés au Moyen Age, Comité pour l'histoire économique et monétaire de la France, Paris, 1994.

Depeyrot, Georges, Histoire de la monnaie, t. II, Du quatorzième siècle au seizième siècle, Wetteren (Belgique), éd. Monéta, 1995.

Dumas, Françoise, Le monnayage des ducs de Bourgogne, Louvain-la-Neuve, 1985.

Duplessy, Jean, Les monnaies royales françaises, t. I, Paris-Maastricht, 1988.

Feller, Laurent, Faux-monnayeurs et fausses monnaies en France à la fin du Moyen Age, Paris, 1986.

Fournial, Etienne, Histoire monétaire de l' Occident médiéval, Paris, 1970.

Grierson, Philip, Monnaies et monnayages : introduction à la numismatique, Paris, 1988.

Journal du Bourgeois de Paris, Paris, éd. C. Beaune, 1990, p. 169 (305 et 306) et p. 170 (310).

Lafaurie, Jean, Les monnaies des rois de France. Hugues Capet à Louis XII, Paris-Bâle, 1951.

Lardin, Philippe, «Un manœuvre privilégié, le valet de la fabrique de la cathédrale de Rouen, à la fin du Moyen Age», dans Chapitres et cathédrales en Normandie, textes recueillis par S. Lemagnen et P. Manneville, Actes du XXX ${ }^{e}$ Congrès des sociétés historiques et archéologiques de Normandie (Bayeux, 16-20 octobre 1996), Caen, Musée de Normandie, Annales de Normandie-Série des Congrès des sociétés historiques et archéologiques, vol. 2, pp. 361-373.

Lardin, Philippe, «Le niveau de vie des ouvriers du bâtiment en Normandie orientale dans la seconde moitié du XV e siècle», Actes du Colloque de Spa (21-25 octobre 1998) édités par Jean-Pierre Sosson, Claude Thiry, Sandrine Thonon, Tania van Hamelryck, Université catholique de Louvain, Academia-Bruylant, 1999, pp. 141-173.

Lesage, J., «La circulation monétaire en France dans la seconde moitié du $\mathrm{XV}^{\mathrm{e}}$ siècle», Annales E.S.C., 1948, 3, pp. 304-317.

Menjot, Denis, «Les métiers en Castille au Bas Moyen Age : une approche des "vécus socio-économiques"», dans Les métiers au Moyen Age. Aspects économiques et sociaux, Actes du Colloque 
international de Louvain-la-Neuve (7-9 octobre 1993), textes édités par Lambrechts Pascale et Sosson, Jean-Pierre, Louvain-la-Neuve, 1994, p. 222-226.

Miskimin, Harry A., Money and Power in Fifteenth-Century France, New Haven, 1984.

Moesgaard, J.-C., et Cliquet, D., Les dessous des sous, Catalogue d'exposition, Evreux, 1992.

Moesgaard, J.-C., La politique monétaire des rois lancastriens en Haute-Normandie de 1417/19 à 1449/50. Une étude de la situation monétaire, Mémoire de maîtrise, dir. Kai Horby, Université de Copenhague, juin 1992.

Mollat, Michel, Le commerce maritime normand au XV siècle et au début du XVI siècle, Paris, 1953, p. 28.

La moneta nelle' economia europea secc. XIII-XVIII, Prato, 1983, (7emaine d'étude, 1975, de 1'Istituto internazionale di storia economica «F. Datini»).

Ordonnances des rois de France, t. XI.

Poey d'Avant, F., Monnaies féodales de France, Paris, 1852-1862, réimpression Graz, 1961, rééd. Paris, G. Depeyrot, 1995.

Saulcy (de), Félicien, Histoire numismatique de Henry $V$ et Henry VI, rois d'Angleterre, pendant qu'ils ont régné en France, Paris, 1878, p. 7.

Spufford, Peter, Money and its Use in Medieval Europe, Cambridge, 1988.

Van Werveke, Hans, «Monnaie de compte et monnaie réelle», Revue belge de philologie et d' histoire, XIII, 1934. 